\title{
The Clinical Effects of Febuxostat Alone or Combined with Arthroscopic Surgery for Gout: A Single-Center Retrospective Study
}

\author{
Zhen Gong' \\ Li Xia' \\ Rune $X u^{\prime}$ \\ Min Luo' \\ Hongxiang Deng' \\ Zhiping Kang' \\ Leping Liu' \\ Yaqing Liu' \\ Fangjie Zhang (iD ${ }^{2}$ \\ Jian Shi ${ }^{1}$
}

'Department of Rheumatology, Yiyang Central Hospital, Yiyang, Hunan, People's Republic of China; ${ }^{2}$ Department of Emergency Medicine, Xiangya Hospital, Central South University, Changsha, Hunan, People's Republic of China
Correspondence: Fangjie Zhang Department of Emergency Medicine, Xiangya Hospital, Central South University, No. 87 Xiangya Road,

Changsha, Hunan, 410008, People's

Republic of China

Tel +86 I5I I6256248

Email zhangfj@csu.edu.cn

Jian Shi

Department of Rheumatology, Yiyang

Central Hospital, No. 1 I8 Kangfu North

Road, Yiyang, Hunan, 413000, People's

Republic of China

$\mathrm{Tel}+86$ I860737I607

Email 408739063@qq.com
Purpose: The purpose of the study was to retrospectively analyze the effects of febuxostat combined with arthroscopic debridement of monosodium urate crystal deposition and febuxostat treatment alone on uric acid levels and acute flares in gout patients.

Patients and Methods: We retrospectively analyzed gout patients who were treated from February 2016 to December 2020. Patients were divided into a control group (febuxostat treatment alone) or a combined group (febuxostat combined with arthroscopic surgery). We recorded and analyzed clinical data including age, sex, body mass index, comorbidities, lesion affected joints, acute flare times, medications history, febuxostat side effects, arthroscopic complications, and serum creatinine and uric acid levels changes.

Results: There were 80 patients in the control group and 93 patients in the combined group. At the beginning of treatment, the combined group had significantly higher disease severity (higher serum uric acid levels and more acute gout flare times). Arthroscopy was performed in 61 knees and 38 ankles, and 86 joints showed crystals deposition. Compared with baseline, follow-up results showed that serum creatinine significantly decreased in the combined group, and serum uric acid and acute gout flare times significantly decreased in both groups (all $p<$ 0.05). In the comparison between the two groups at the follow-up endpoint, acute gout flare times did not differ significantly $(p>0.05)$, however, serum creatinine and uric acid levels were lower in the combined group compared with those in the control group ( $p \mathrm{~s}<0.05)$.

Conclusion: Febuxostat combined with arthroscopic debridement of monosodium urate crystal deposition or tophi had a superior effect on lowering uric acid levels and acute flare times in gout patients than did febuxostat alone.

Keywords: arthroscopy, febuxostat, crystal deposition, tophi, gout

\section{Introduction}

Gout is a disease involving monosodium urate crystal deposition in tissues, which results in painful recurrent flares and tissue damage. ${ }^{1}$ Biochemically, gout results from the concentration of urate in the extracellular fluid becoming saturated, which appears as hyperuricemia in the blood, Uric acid is the final metabolite of endogenous and dietary purine metabolism, and urate is largely present as monosodium urate, with a low solubility limit of approximately $380 \mu \mathrm{mol} / \mathrm{L}$. When urate concentrations exceed $380 \mu \mathrm{mol} / \mathrm{L}$, the risk of monosodium urate crystal formation and precipitation increases. ${ }^{2}$ The progression of gout can be defined by four pathophysiological stages: 1) the asymptomatic hyperuricemia stage, which is hyperuricemia without crystal deposition; 2) crystal deposition without symptomatic gout; 3) acute 
gout flares (acute gouty arthritis); and 4) chronic tophaceous, which is advanced gout characterized by tophi, chronic arthritis, and radiographic damage. ${ }^{3}$ In tissues, monosodium urate crystal deposition depends on several factors: local concentration of urate (local concentration of urate in joint fluids associated with the articular hydration state), temperature, $\mathrm{pH}$, concentration of cations, and presence of extracellular matrix proteins, such as proteoglycans, collagens, and chondroitin sulfates. ${ }^{4}$ At $37^{\circ} \mathrm{C}$ and with a $\mathrm{pH}$ of 7.4 , levels of uric acid above $408 \mu \mathrm{mol} / \mathrm{L}$ result in crystal formation and aggregation ${ }^{5}$ in peripheral joints, and with lower tissue $\mathrm{pH}$ and temperature, monosodium urate crystallization can occur at urate concentrations lower than $408 \mu \mathrm{mol} / \mathrm{L}$ (eg, at $35^{\circ} \mathrm{C}$, in-vitro crystallization occurs at $360 \mu \mathrm{mol} / \mathrm{L}$ ). ${ }^{3}$

The key to treating gout is using long-term uratelowering therapy, which leads to the dissolution of monosodium urate crystals and ultimately results in the prevention of gout flares and tophi and improved quality of life. ${ }^{1,6}$ Febuxostat is a xanthine oxidase (XO) inhibitor that was approved for use as a treatment for hyperuricemia of gout in 2009. ${ }^{7,8}$ Febuxostat is non-inferior to allopurinol therapy with respect to the primary cardiovascular endpoint, and its long-term use is not associated with an increased risk of death or serious adverse events compared with allopurinol. ${ }^{9}$ The 2020 American College of Rheumatology guidelines for the management of gout strongly recommends either allopurinol or febuxostat for patients with moderate-to-severe chronic kidney disease (CKD; stage $\geq 3$ ). ${ }^{10}$ In China, allopurinol, febuxostat, or benzbromarone are recommended as first-line drugs for long-term urate-lowering therapy. ${ }^{11}$ However, in clinical settings, many patients do not receive regular treatment for hyperuricemia or during the early stage of gout, and crystal deposition and tophi develop in those with poorly treated or uncontrolled chronic gout.

Crystal deposition in the joints occurs as an acute flare and leads to joint dysfunction and joint damage. Surgical excision of tophi is uncommon and is most removal of subcutaneous tophi, ${ }^{12}$ Few arthroscopic studies have been conducted on the treatment of intra-articular tophi or crystals. A few English language studies have reported the use of arthroscopy to remove intra-articular crystals. ${ }^{13,14}$ However, in China, several studies have reported that arthroscopy is useful for the treatment of acute gouty arthritis. ${ }^{15,16}$ Since 2016, our hospital has been performing arthroscopic surgeries to treat gout. Thus, the purpose of the present study was to retrospectively analyze the clinical effects of febuxostat combined with arthroscopic debridement of monosodium urate crystal deposition and febuxostat treatment alone on uric acid levels and acute flares in gout patients.

\section{Materials and Methods}

The protocol of arthroscopic surgery treatment for gout was approved by the ethics committee (MR-43-20005173) of the Medical Research Registration Information System of the National Health Commission of the People's Republic of China, and the current retrospective study protocol was approved by the ethics committee (No.2021-35) of Yiyang Central Hospital (Yiyang city, Hunan province, China) and complied with the Declaration of Helsinki. The data in the present study were retrospective and anonymous; therefore, the requirement for patient informed consent was waived.

\section{Study Population}

We retrospectively analyzed gout patients who were treated in the Department of Rheumatology, Yiyang Central Hospital, from February 2016 to December 2020. The inclusion criteria of the study were confirmed diagnosis of gout and received febuxostat treatment alone or febuxostat combined with arthroscopic debridement of monosodium urate crystal deposition (regardless of operation times). All patients met clinical diagnostic criteria for gout according to the 2015 gout classification criteria of the American College of Rheumatology/European League Against Rheumatism. ${ }^{17}$ Patients were divided into a control group (gout patients who insisted on taking febuxostat treatment) and a combined group (gout patients who received arthroscopic debridement of monosodium urate crystal deposition or tophi and insisted on taking febuxostat treatment). Patients in both groups received febuxostat using a "titrated increment" method, whereby the dosage was gradually increased from $10 \mathrm{mg} / \mathrm{d}$ (4 weeks), $20 \mathrm{mg} / \mathrm{d}$ (4 weeks), to $40 \mathrm{mg} / \mathrm{d}$ (persisted to the final follow-up), and colchicine $0.5 \mathrm{mg} / \mathrm{d}$ was given simultaneously in the first 4 weeks for a creatinine clearing rate $>50 \mathrm{~mL} / \mathrm{min}$. During the study period, when patients experienced an acute gout flare, colchicine and nonsteroidal anti-inflammatory drugs (NSAIDs) were provided as a first choice to control acute gout flares under the guidance of doctors. Gout flares were defined as episodes of joint pain with acute onset and maximum pain, usually within $24 \mathrm{~h} .{ }^{18}$ For patients with an acute gout flare who failed to respond to colchicine and NSAIDs, shortterm glucocorticoid therapy was administered. 


\section{Arthroscopic Surgery}

Indications for arthroscopic surgery were refractory gout, acute gouty arthritis not relieved by colchicine and NSAIDs, and chronic gouty arthritis with severe joint pain. Arthroscopic surgery contraindications were history of knee surgery or severe trauma, local skin infection of the joint, comorbidities, such as abnormal coagulation function, severe infection, or thrombosis before surgery, and mental illness.

All joints that underwent arthroscopic surgery were knee or ankle joints. During the operation, synovial fluid was first punctured, suctioned, and sent for laboratory examination, and the joint cavity was inspected. A blunt spatula or curette was used to remove the urate crystals deposited in the meniscus, ligaments, synovium, joint capsule wall, and cartilage surface. If crystals had adhered close to healthy tissue and were difficult to peel off, they were not removed to avoid damaging the healthy joint structure. Furthermore, if the synovial membrane had obvious hyperemia, we also did not treat it to avoid excessive bleeding during or after surgery. During the operation, a large amount of saline was used to wash out the joint cavity. After the operation, one sodium hyaluronate (20mg/2mL, Bausch\&Lomb Freda Co., Ltd., Shandong, China) and one compound betamethasone Injection $(5 \mathrm{mg}$ $+2 \mathrm{mg} / 1 \mathrm{~mL}$, Schering-Plough Pharmaceutical Co., Ltd. Shanghai,China) were injected into the joint cavity. The severity of monosodium urate crystal deposition in the joint was scored on a scale from 0-3 (grade 0: no urate crystal deposition; grade 1: scattered distribution of crystal deposition; grade 2: multiple crystal depositions in $<50 \%$ of the affected articular cartilage and soft tissues areas; and grade 3: multiple crystal depositions of $>50 \%$ of the articular cartilage and soft tissue areas, crystal erosion of the articular cartilage and subchondral bone, or "snowstorm sign" under arthroscopy). ${ }^{19}$ All arthroscopic surgeries were performed by the author, Zhen Gong.

\section{Data Collection}

The following data were collected from patients: age, gender, body mass index (BMI), comorbidities, lesion affected joints, acute flare times, medications used before receiving febuxostat, febuxostat side effects, arthroscopic complications, and serum creatinine and uric acid levels at the time of admission to our hospital and the final follow-up.

\section{Statistical Analysis}

Data were analyzed using GraphPad Prism 5 (GraphPad Software, Inc. La Jolla, CA,USA) for evaluation. Results are expressed as means \pm standard deviations (SDs).
Continuous variables were compared using Student's $t$-test for normally distributed measures and the Mann-Whitney $U$-test for non-normally distributed measures. Comparisons of enumeration data between groups were performed using chi-square or Fisher's exact tests, as appropriate. A $p<0.05$ was considered statistically significant.

\section{Results \\ Baseline Measures of the Control and Combined Groups}

A total of 226 patients received febuxostat treatment alone or febuxostat combined with arthroscopic debridement of monosodium urate crystal deposition, and 173 patients completed follow-up. There were 80 patients (78 male and 2 female) in the control group (ie, patients who received febuxostat treatment), which had an average age of 59.3 years, and there were 93 patients (all male) in the combined group (ie, patients who received arthroscopic debridement and febuxostat treatment), which had an average age of 48.4 years (Table 1). As shown in Table 1, the combined group had a higher BMI than the control group $(p<0.05)$, whereas the number of patients who drank alcohol or smoked, comorbidities, and illness duration did not differ significantly (all $p>0.05$ ). The combined group had more elbow and knee joints affected by gout than did the control group $(p s<0.05)$, whereas there were no significant differences in the number of shoulder, wrist, hand, ankle, or foot joints between the two groups (all $p>0.05$; Table 1 ).

Furthermore, results revealed that the combined group had a higher serum uric acid level and more acute gout flares per patient at the time of beginning receiving treatment than did the control group $(p s<0.05)$. However, serum creatinine level was lower in the combined group than in the control group ( $p<0.05$; Table 1). For the comparison of the medication use between the two groups, the combined group used more allopurinol, glucocorticoids, and NSAIDs than did the control group (all $p<0.05$ ), whereas the use of colchicines, benzbromarone, febuxostat, and diacerein were not significantly different (all $p>0.05$ )

\section{Follow-Up Results Between the Two Groups}

Table 2 shows the follow-up results. The follow-up times of the control and combined groups were $31.56 \pm 15.52$ months and $29.03 \pm 17.44$ months, respectively, and did not differ significantly ( $p=0.3183)$. Arthroscopy surgery was performed in 61 knees and 38 ankles in the 93 patients 
Table I Baseline of Control Group and Combined Patients

\begin{tabular}{|c|c|c|c|}
\hline Characteristics & Control Group $(n=80)$ & Combined Group $(n=93)$ & $P$ value \\
\hline Age & $59.25 \pm 16.97$ & $48.35 \pm 14.40$ & 0.0001 \\
\hline Sex (male) & 78 & 93 & 0.2124 \\
\hline Body mass index(BMI) & $24.27 \pm 3.335$ & $25.64 \pm 3.429$ & 0.0089 \\
\hline Drinking & 25 & 35 & 0.4249 \\
\hline Smoking & 32 & 41 & 0.6444 \\
\hline \multicolumn{4}{|l|}{ Comorbidity } \\
\hline Hypertension & 28 & 43 & 0.1634 \\
\hline Coronary Heart Disease & 6 & 2 & 0.1463 \\
\hline Diabetes & 7 & 8 & 1.0000 \\
\hline Stroke & 2 & I & 0.5964 \\
\hline Hyperlipidemia & 2 & 0 & 0.2124 \\
\hline Lung cancer & I & 0 & 0.4624 \\
\hline COPD & I & 0 & 0.4624 \\
\hline Kidney stones & I & 0 & 0.4624 \\
\hline Rheumatoid Arthritis & 0 & I & 1.0000 \\
\hline Serum Creatinine $(\mu \mathrm{mol} / \mathrm{L})$ & $120.3 \pm 75.90$ & $90.31 \pm 31.07$ & 0.0006 \\
\hline Serum Uric acid $(\mu \mathrm{mol} / \mathrm{L})$ & $469.5 \pm 147.3$ & $526.7 \pm 115.8$ & 0.0050 \\
\hline Illness length time (years) & $7.988 \pm 5.960$ & $7.222 \pm 5.432$ & 0.3779 \\
\hline Acute gout flares per patient before treatment I year (total) & $5.713 \pm 4.792(457)$ & $7.172 \pm 4.615(667)$ & 0.0431 \\
\hline \multicolumn{4}{|l|}{ Medications before the use of febuxostat } \\
\hline Colchicine & 32 & 48 & 0.1686 \\
\hline Benzbromarone & 5 & 8 & 0.7737 \\
\hline Allopurinol & 3 & 24 & 0.0001 \\
\hline Febuxostat & 6 & 14 & 0.1544 \\
\hline Diacerein & I & 4 & 0.3749 \\
\hline Glucocorticoid & 14 & 30 & 0.0352 \\
\hline NSAIDs & 38 & 67 & 0.0011 \\
\hline \multicolumn{4}{|l|}{ Lesions affect joints (no hip) } \\
\hline Shoulder & 11 & 11 & 1.0000 \\
\hline Elbow & 9 & 24 & 0.0100 \\
\hline Wrist & 10 & 17 & 0.2316 \\
\hline Hand & 27 & 33 & 0.8872 \\
\hline Knee & 44 & 109 & 0.0001 \\
\hline Ankle & 48 & 58 & 0.8163 \\
\hline Foot & 65 & 115 & 0.0001 \\
\hline
\end{tabular}

in the combined group. Twelve joints had no significant crystal deposition under arthroscopy surgery, which were classified as grade 0 . Twenty-two joints were classified as grade 1, 20 joints were classified as grade 2, and 45 joints were classified as grade 3 . The typical changes under arthroscopy are shown in Figure 1. For postoperative complications of arthroscopic surgery, two patients had minor local skin infection of the incision, and two patients had joint effusion and pain.
We also recorded the side effects of febuxostat in the two groups. Incidence of abnormal liver function, nausea, joint pain, muscle ache, and rash were not significantly different between the two groups (all $p>0.05$ ), as shown in Table 2 . Acute gout flares per patient during the follow-up period were also not significantly different between the two groups $(p>0.05)$, as shown in Table 2. Serum creatinine and uric acid levels were lower in the combined group compared with those in the control group ( $p$ s $<0.05$; Table 2). 
Table 2 Follow-Up Results of Control Group and Combined Group Patients

\begin{tabular}{|c|c|c|c|}
\hline Results & Control Group & Combined Group & $P$ value \\
\hline Follow-up time (months) & $31.56 \pm 15.52$ & $29.03 \pm 17.44$ & 0.3183 \\
\hline \multicolumn{4}{|l|}{ Arthroscopic treatment joint numbers } \\
\hline Knee & & 61 & \\
\hline Ankle & & 38 & \\
\hline \multicolumn{4}{|l|}{ The severity of crystal deposition in joint } \\
\hline 0 & & 12 & \\
\hline 1 & & 22 & \\
\hline 2 & & 20 & \\
\hline 3 & & 45 & \\
\hline \multicolumn{4}{|l|}{ Arthroscopic complications } \\
\hline Minor local infection of the incision & & 2 & \\
\hline Joint effusion, pain & & 2 & \\
\hline \multicolumn{4}{|l|}{ Febuxostat side effect } \\
\hline Abnormal liver function & 6 & 8 & 1.0000 \\
\hline Nausea & 3 & 2 & 0.6634 \\
\hline Joint pain & 1 & 3 & 0.6249 \\
\hline Muscle ache & 2 & I & 0.5964 \\
\hline Rash & 0 & I & 1.0000 \\
\hline Total & 12 & 14 & \\
\hline Acute gout flares per patient during follow-up period & $2.713 \pm 2.246$ & $2.87 I \pm 2.950$ & 0.6952 \\
\hline Serum Creatinine $(\mu \mathrm{mol} / \mathrm{L})$ & $109.5 \pm 69.40$ & $79.68 \pm 20.32$ & 0.0001 \\
\hline Serum Uric acid ( $\mu \mathrm{mol} / \mathrm{L})$ & $371.8 \pm 91.80$ & $341.1 \pm 71.75$ & 0.0167 \\
\hline
\end{tabular}

\section{Comparison Between Before and After Treatment for Each Group}

The comparisons between before and after treatment in each group showed that serum creatinine did not significantly decrease in the control group ( $p=0.3639$, Figure 2$)$. In the combined group, serum creatinine significantly decreased from baseline to follow-up ( $p=0.0063$, Figure 2). Serum uric acid showed a significant decrease in both the control and combined groups from baseline to follow-up ( $p$ s $<0.0001$; Figure 3). Furthermore, our results indicated that acute gout flares per patient significantly decreased in both the control and combined groups from baseline to follow-up ( $p s<0.0001$; Figure 4$)$.

\section{Discussion}

Gout is an extremely common chronic rheumatic disease that presents with an intermittent flaring condition. The lower limbs (foot, ankle, and knee) are preferentially affected, especially the first metatarsophalangeal joint. ${ }^{19}$ Urate lowering is achieved via two primary pharmacological mechanisms: decreased synthesis of uric acid and increased renal excretion of uric acid. ${ }^{20}$ Long-term uratelowering therapy using medications, such as allopurinol or febuxostat, leads to the dissolution of monosodium urate crystals. ${ }^{1}$ The American College of Rheumatology guidelines recommend a serum urate target of less than 360 $\mu \mathrm{mol} / \mathrm{L}$ for patients on urate-lowering therapy. ${ }^{10}$ For patients with frequent polyarticular flares, numerous tophi, and joint damage, current treatment is intensive individualized pharmacological management of a combination of urate-lowering therapy and anti-inflammatory agents. ${ }^{21}$ However, there are currently no guidelines and only a few studies on the surgical treatment of gout due to deposition of tophi or crystals in the joints.

In our study, there were 2 females and 171 male patients in the control group, which is in line with a previous study showing that in China, the prevalence of hyperuricemia and gout is significantly higher in men than in women. ${ }^{22}$ Furthermore, the combined group were younger but had higher BMI, higher serum uric acid levels at the time of beginning receiving treatment, and more acute gout flares per patient during the period time of 1 year before 
A

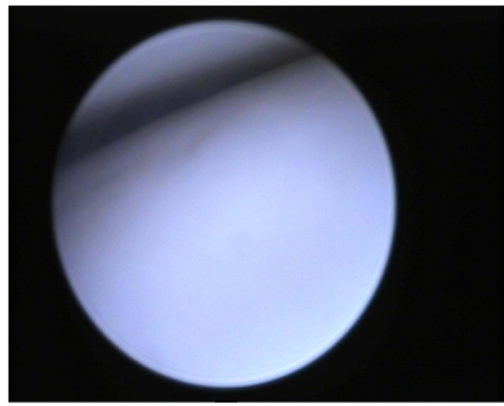

D

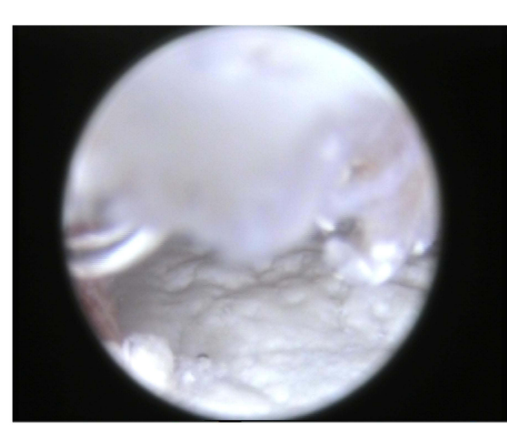

Grade 3

G

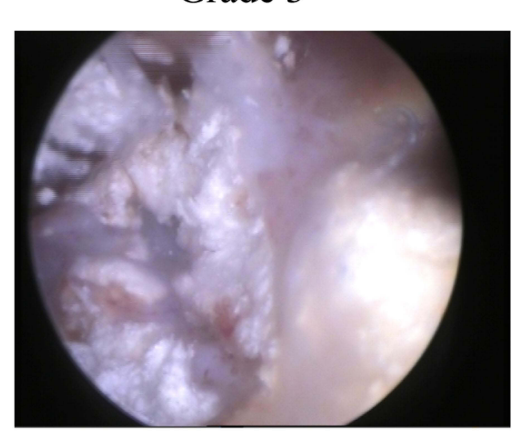

Grade 3
B

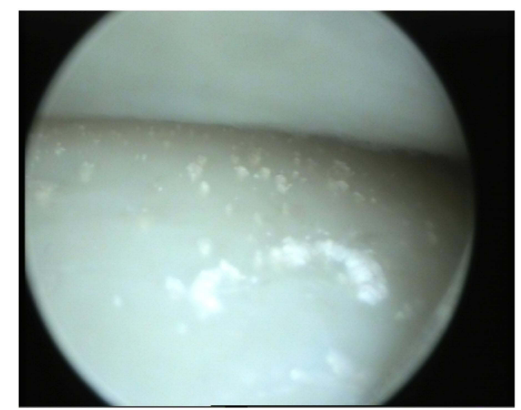

Grade 1

E

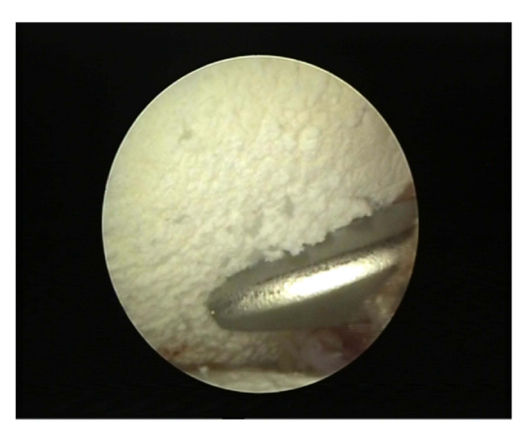

Grade 3

H

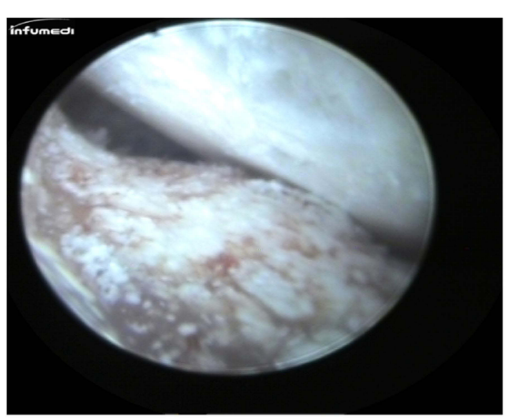

Grade 3
C

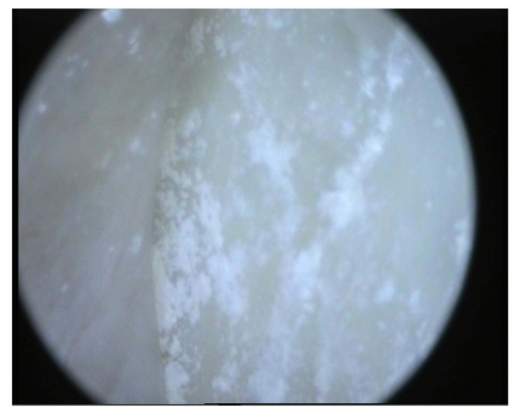

Grade 2

F

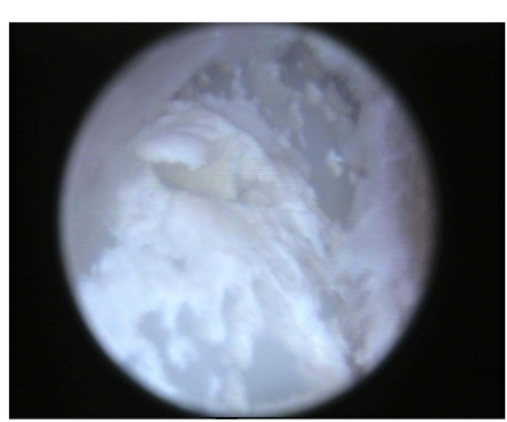

Grade 3

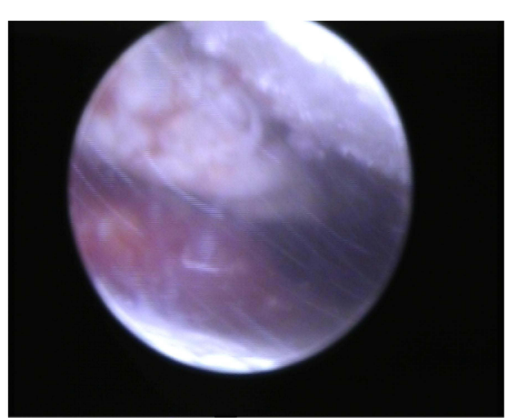

Snow storm sign

Figure I Arthroscopy showed the monosodium urate crystal deposition or tophi in joints. (A) No obvious crystal deposition. (B) Distribution of crystals is scattered. (C) Multiple crystal depositions in $<50 \%$ of the affected articular cartilage and soft tissues. (D-H) Multiple crystal depositions in $>50 \%$ of the articular cartilage and soft tissues, crystal erosion of the articular cartilage, or large tophi. (I) The snow storm sign.

treatment, and higher use of allopurinol, glucocorticoids, and NSAIDs than did the control group. In addition, the elbows, knees, and feet were more affected by gout in the combined group than in the control group. However, serum creatinine levels in the control group were higher than those in the combined group. The data at baseline indicated that the combined group had greater disease severity than the control group, except for serum creatinine levels. An observational study that included 9754 Chinese gout patients showed that male sex, obesity, and elevated serum uric acid were independent predictors of early-onset gout in young gout patients. ${ }^{23}$ The discrepancies in findings may be associated with differences in inclusion criteria; because our study was not a randomized double-blind controlled trial but a retrospective study, many patients voluntarily requested arthroscopic surgery because of long-term pain from recurrent acute gouty arthritis flares. Thus, uric acid levels, the number of acute arthritis flares, and glucocorticoid and analgesic use in these patients would be expected to be higher than in the previous study, which was confirmed by our data. Our results also indicated that serum creatinine levels in the control group were higher than those in the combined group, which may be attributed to doctor and patient concerns that arthroscopic surgery may aggravate renal function damage and thus, arthroscopy was not performed in patients with high serum creatinine. 


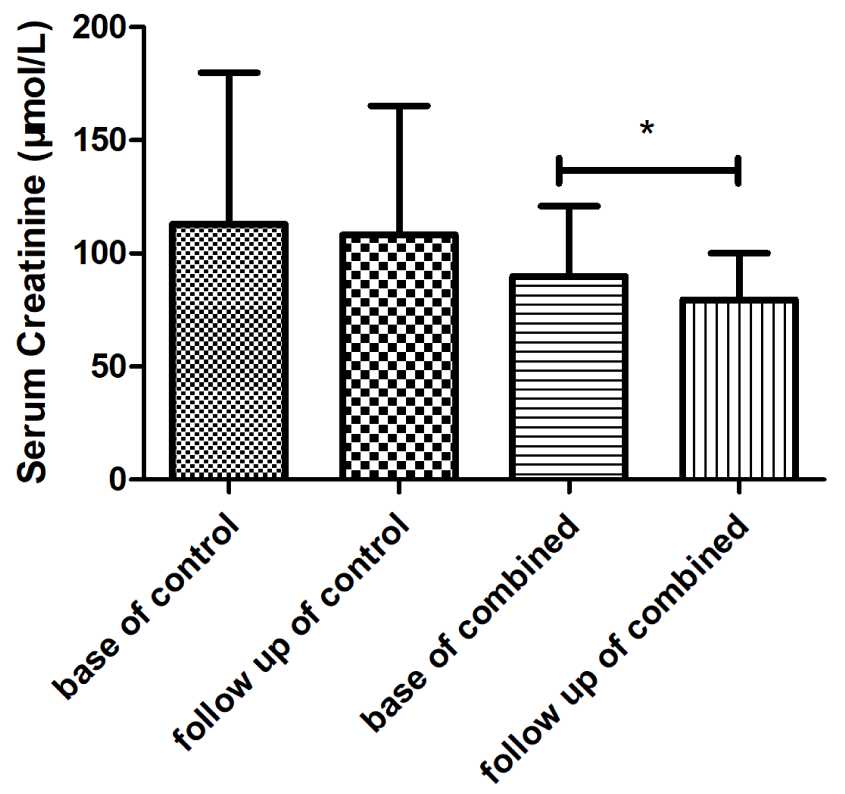

Figure 2 Mean blood creatinine level change between baseline and follow-up time in the control and combined groups. ${ }^{*} p<0.05$.
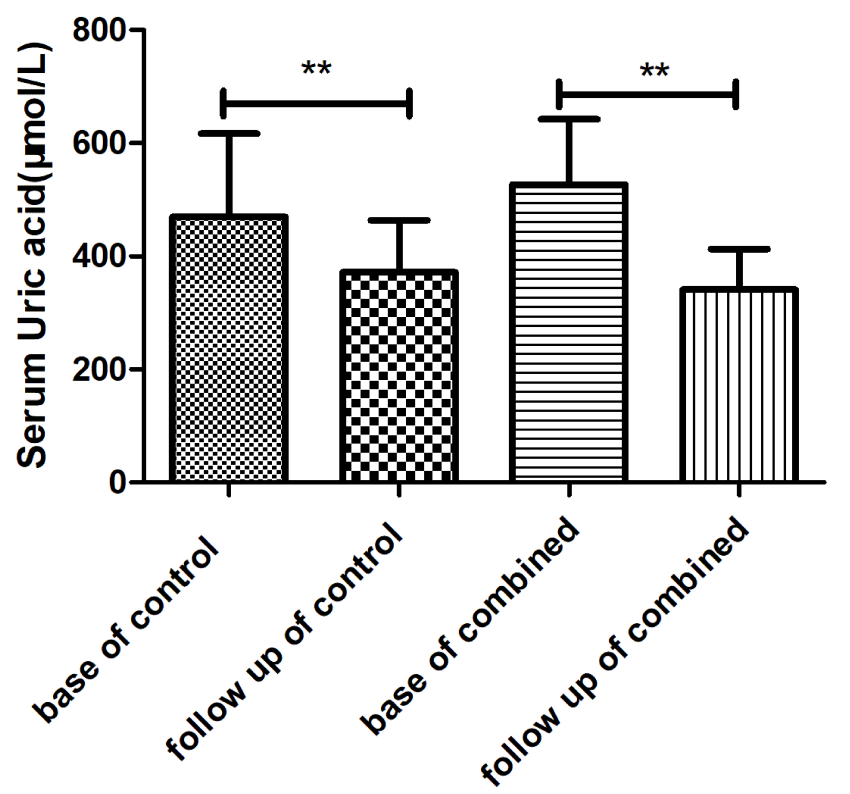

Figure 3 Mean blood uric acid change between baseline and follow-up time in the control and combined groups. $* * p<0.0001$.

Our study showed that serum creatinine, uric acid, and the number of acute flares decreased significantly in the combined group, which confirmed that febuxostat combined with arthroscopic debridement of monosodium urate crystal deposition or tophi was more effective than febuxostat alone. Moreover, we confirmed that long-term use of febuxostat has few adverse effects. A previous study confirmed that febuxostat is non-inferior to allopurinol therapy

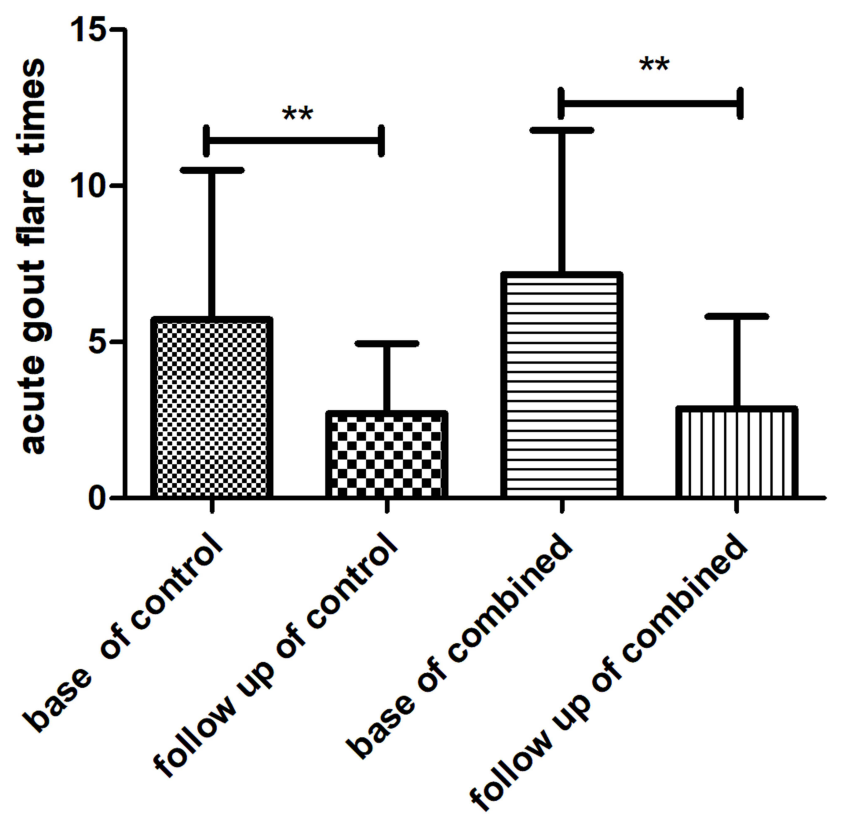

Figure 4 Mean number of acute gout flares between baseline and follow-up time in the control and combined groups. $* * p<0.0001$.

and that long-term use of febuxostat is not related to an increased risk of death or serious adverse events. ${ }^{9}$ Febuxostat has been shown to improve synovitis and reduce the incidence of gout flares in patients with early gout, and it does not result in any notable changes in joint erosion over 2 years. ${ }^{24}$ However, another study revealed that daily pain, recurrent flares, and adverse effects with the initiation of febuxostat during an acute gout flare did not differ significantly from those of controls, although febuxostat treatment significantly decreased uric acid levels during the early stage of gout. ${ }^{25}$ In our study, most arthroscopic joints of patients in the combined group $(88.78 \%, 87 / 98$ joints) had monosodium urate crystal deposition or tophi under arthroscopy, and the crystals in the joint could be removed completely. Drug treatments require a longer period to slowly dissolve the crystals. However, the mechanisms underlying intraarticular monosodium urate crystal deposition or tophi formation remain poorly understood, although hyperuricemia and initial abnormalities in joint structure are known to play an important role. ${ }^{26}$ We did not re-examine the operated joints to assess further recrystallized deposits, and it is unclear whether uric acid levels decreased further following arthroscopic debridement of crystals. However, for the treatment of gouty arthritis, arthroscopy provides only local joint treatment and cannot completely correct abnormal purine metabolism. Therefore, standard comprehensive treatment of 
gouty arthritis is necessary, which includes diet adjustment, weight control, regular work and rest, and drug treatment. Many patients who were lost to follow-up in this study did not comply with the prescribed urate-lowering therapy, which resulted in frequent flares of gouty arthritis and loss in the willingness of patients to continue treatment.

\section{Limitations}

In this study, we recorded the total number of gouty arthritis flares in each patient but did not distinguish between flares of the surgical and non-surgical joints. Because the arthroscopic surgeons were rheumatologists, arthroscopic surgeries were only performed in the knee and ankle joints and not in other complex joints, such as small joints and those that require technically demanding surgeries. Furthermore, we only performed simple washing of tophi crystals during arthroscopic surgery and did not perform surgical operations on other joint structures. Nevertheless, our study findings may encourage arthroscopic surgeons to actively treat severe gout.

\section{Conclusion}

Febuxostat combined with arthroscopic debridement of monosodium urate crystal deposition or tophi was more effective for reducing uric acid levels and the number of acute flares in gout patients than febuxostat alone.

\section{Data Sharing Statement}

All data generated or analyzed during this study are included in the manuscript.

\section{Consent for Publication}

All authors have read and approved the manuscript. The data in the present study were retrospective and anonymous, and the requirement for patients informed consent was therefore waived.

\section{Funding}

This work was supported by the National Natural Science Foundation of China (No.81501923), the Science and Technology Innovation Program of Hunan Province (No.2018SK52206) and the Rui E (Ruiyi) Emergency Medical Research Special Funding Project (No. R2019007).

\section{Disclosure}

The authors declare that there are no conflicts of interest.

\section{References}

1. Dalbeth N, Gosling AL, Gaffo A, et al. Gout. Lancet. 2021;397 (10287):1843-1855. doi:10.1016/S0140-6736(21)00569-9

2. Richette P, Bardin T. Gout. Lancet. 2010;375(9711):318-328. doi:10.1016/S0140-6736(09)60883-7

3. Dalbeth N, Merriman TR, Stamp LK. Gout. Lancet. 2016;388(10 055):2039-2052. doi:10.1016/S0140-6736(16)00346-9

4. Choi HK, Mount DB, Reginato AM. Pathogenesis of gout. Ann Intern Med. 2005;143(7):499-516. doi:10.7326/0003-4819-143-7-20 0510040-00009

5. Ruoff G, Edwards NL. Overview of serum uric acid treatment targets in gout: why less than $6 \mathrm{mg} / \mathrm{dL}$ ? Postgrad Med. 2016;128 (7):706-715. doi:10.1080/00325481.2016.1221732

6. Singh JV, Bedi P, Singh H, et al. Xanthine oxidase inhibitors: patent landscape and clinical development (2015-2020). Expert Opin Ther Pat. 2020;30(10):769-780. doi:10.1080/13543776.2020.1811233

7. Kumar R, Darpan SS, Sharma S, et al. Xanthine oxidase inhibitors: a patent survey. Expert Opin Ther Pat. 2011;21(7):1071-1108. doi: $10.1517 / 13543776.2011 .577417$

8. Ojha R, Singh J, Ojha A, et al. An updated patent review: xanthine oxidase inhibitors for the treatment of hyperuricemia and gout (20112015). Expert Opin Ther Pat. 2017;27(3):311-345. doi:10.1080/135 43776.2017.1261111

9. Mackenzie IS, Ford I, Nuki G, et al. Long-term cardiovascular safety of febuxostat compared with allopurinol in patients with gout (FAST): a multicentre, prospective, randomised, open-label, non-inferiority trial. Lancet. 2020;396(10264):1745-1757. doi:10.1016/S0140-6736 (20)32234-0

10. Fitzgerald JD, Dalbeth N, Mikuls T, et al. American college of rheumatology guideline for the management of gout. Arthritis Rheumatol. 2020;72(6):879-895. doi:10.1002/art.41247

11. Rheumatology Branch of Chinese Medical Association. China gout clinical practice guideline. Zhonghua Nei Ke Za Zhi. 2016;55 (11):892-899.

12. Carcione J, Bodofsky S, LaMoreaux B, et al. Beyond medical treatment: surgical treatment of gout. Curr Rheumatol Rep. 2020;23(1):1. doi:10.1007/s11926-020-00969-6

13. Steinmetz RG, Maxted M, Rowles D. Arthroscopic management of intra-articular tophaceous gout of the knee: a case report and review of the literature. J Orthop Case Rep. 2018;8(2):86-89.

14. Aoki T, Tensho K, Shimodaira H, et al. Intrameniscal gouty tophi in the knee: a case report. JBJS Case Connect. 2015;5(3):e74. doi:10.21 06/JBJS.CC.N.00191

15. Li HL, Li SY, Li CB, et al. Clinical observation of arthroscopic debridement for acute gouty arthritis of the ankle. Zhongguo $G u$ Shang. 2016;29(3):258-260.

16. Pan F, Li Q, Tang X, et al. Method and effectiveness of arthroscopic debridement for treating gouty arthritis of the knee. Zhongguo Xiu Fu Chong Jian Wai Ke Za Zhi. 2011;25(8):937-940.

17. Neogi T, Jansen TL, Dalbeth N, et al. Gout classification criteria: an American college of rheumatology/European league against rheumatism collaborative initiative. Arthritis Rheumatol. 2015;67(10):25 57-2568. doi:10.1002/art.39254

18. Neogi T. Gout. Ann Intern Med. 2016;165(1):ITC1-16. doi:10.7326/ AITC201607050

19. Lamers-Karnebeek FB, Van Riel PL, Jansen TL. Additive value for ultrasonographic signal in a screening algorithm for patients presenting with acute mono-/oligoarthritis in whom gout is suspected. Clin Rheumatol. 2014;33(4):555-559. doi:10.1007/s10067-014-2505-6

20. Wilson L, Saseen JJ. Gouty arthritis: a review of acute management and prevention. Pharmacotherapy. 2016;36(8):906-922. doi:10.1002/ phar.1788

21. Pascual E, Andres M, Vazquez-Mellado J, et al. Severe gout: strategies and innovations for effective management. Joint Bone Spine. 2017;84(5):541-546. doi:10.1016/j.jbspin.2016.10.004 
22. Miao Z, Li C, Chen Y, et al. Dietary and lifestyle changes associated with high prevalence of hyperuricemia and gout in the Shandong coastal cities of Eastern China. J Rheumatol. 2008;35(9):1859-1864.

23. Gao Q, Cheng X, Merriman TR, et al. Trends in the manifestations of 9754 gout patients in a Chinese clinical center: a 10-year observational study. Joint Bone Spine. 2020:105078. doi:10.1016/j.jbspin.2020.09.010

24. Dalbeth N, Saag KG, Palmer WE, et al. Effects of febuxostat in early gout: a randomized, double-blind, placebo-controlled study. Arthritis Rheumatol. 2017;69(12):2386-2395. doi:10.1002/art.40233
25. Sun R, Lu J, Li H, et al. Evaluation of febuxostat initiation during an acute gout attack: a prospective, randomized clinical trial. Joint Bone Spine. 2020;87(5):461-466. doi:10.1016/j.jbspin. 2020.03.017

26. Desai J, Steiger S, Anders HJ. Molecular pathophysiology of gout. Trends Mol Med. 2017;23(8):756-768. doi:10.1016/j.molmed.2017. 06.005

\section{Publish your work in this journal}

The Journal of Inflammation Research is an international, peerreviewed open-access journal that welcomes laboratory and clinical findings on the molecular basis, cell biology and pharmacology of inflammation including original research, reviews, symposium reports, hypothesis formation and commentaries on: acute/chronic inflammation; mediators of inflammation; cellular processes; molecular mechanisms; pharmacology and novel anti-inflammatory drugs; clinical conditions involving inflammation. The manuscript management system is completely online and includes a very quick and fair peerreview system. Visit http://www.dovepress.com/testimonials.php to read real quotes from published authors.

Submit your manuscript here: https://www.dovepress.com/journal-of-inflammation-research-journal 